Regular Article

\title{
Folding of cytosine-based nucleolipid monolayer by guanine recognition at the air-water interface
}

\author{
Pablo Gómez-Argudo, Eulogia Muñoz, Juan-José Giner-Casares, María-Teresa Martín-Romero, Luis Camacho \\ Institute of Fine Chemistry and Nanochemistry, Department of Physical Chemistry and Applied Thermodynamics, Universidad de Córdoba, Campus Universitario de Rabanales, \\ Edificio Marie Curie, Córdoba E-14014, Spain
}

\section{A R T I C L E I N F O}

Article history

Received in revised form 2 November 2018

Accepted 10 November 2018

Available online $\mathrm{xxx}$

Keywords:

Langmuir monolayer

Cytosine-based nucleolipid

Computer simulations

Complementary base pairing
Received 4 September 2018

\begin{abstract}
A B S T R A C T
Monolayers of a cytosine-based nucleolipid (1,2-dipalmitoyl-sn-glycero-3-(cytidine diphosphate) (ammonium salt), CDP-DG) at basic subphase have been prepared at the air-water interface both in absence and presence of guanine. The formation of the complementary base pairing is demonstrated by combining surface experimental techniques, i.e., surface pressure $(\pi)$-area $(A)$, Brewster angle microscopy (BAM), infrared spectroscopy (PM-IRRAS) and computer simulations. A folding of the cytosine-based nucleolipid molecules forming monolayer at the air-water interface occurs during the guanine recognition as absorbate host and is kept during several compression-expansion processes under set experimental conditions. The specificity between nitrogenous bases has been also registered. Finally, mixed monolayers of CDP-DG and a phospholipid (1,2-dimyristoyl-sn-glycero-3-phosphate (sodium salt), DMPA) has been studied and a molecular segregation of the DMPA molecules has been inferred by the additivity rule.
\end{abstract}

\section{Introduction}

Biorecognition of nucleic acids is a central process in life. Consequently, in the last decades DNA and RNA nanothecnology has attracted great interest from many researchers working in the design of well-defined nanostructures for potential applications. On this framework, to produce new materials, electronic nanodevices, and biosensors novel strategies have been extensively investigated. Self-assembly of cytosine, thymine, adenine, and uracil has been explored on solid surfaces revealing the formation of $1 \mathrm{D}$ and 2D supramolecular nanostructures [1], aggregates highly fluorescent [2]. efficient sensors to distinguish the unique patters of viral nucleic acid/bacterial fragments [3], new nucleic acid-based carrier systems to improve the drug action [4], combination of nucleic acid derivatives with lipids rendering hybrid materials as promising supramolecular materials for biomedical and technological applications [5], fabrication of supramolecular gels formed by nucleic acid-based hydrogels to design particular type of gelators [6], scanning of RNA nano-bio interface by functionalized nanoparticles (NPs) to inquire on the RNA nano-bio interaction [7], or nucleic acids-modified NPs to improve the drug delivery and its efficiency [8].

The performance of most those new nucleic acid-based nanoarchitectures to different applications are ground on the molecular recognition between nucleobases in DNA and RNA. It is a fact that molecular recognition is one of the most significant chemical occurrence not only in supramolecular chemistry [9], but also in biochemical frameworks [10]. Particularly, recognition of nucleobases in DNA and RNA is crucial in genetic transmission and protein expression: DNA makes RNA and RNA makes protein. In such cases, nucleobase pairing occurs because complementary hydrogen bonding. In this context, a stimulus established during the last decades has been the design of artificial recognition systems in solution [11], or interfaces [12]. In the last case, different strategies have been designed to analyse and mimicry of the molecular recognition and differentiation between structurally almost identical molecules as nucleic acids at air-water interface. Thus, quite simple host molecules have been mechanically adapted at the air-water interface and this relatively simple implement has allowed the chiral recognition of amino acids by applying mechanical forces to host monolayers prepared at the air-water interface $[13,14]$. Furthermore, mechanical tuning of host structure for optimization of molecular recognition tenders an appropriate methodology in host-guest chemistry [15].

The Langmuir monolayers at the air-water interface afford unique frame for molecular interactions and accordingly molecular recognition [16]. To construct superlattice materials whose structure is controlled at molecular level, the air-water interface is expected to offer a model environment for preparing films with controlled 2D framework by an appropriate matrix and under external control like surface pressure.

Nucleolipids are functionalized lipids with nucleosides or nucleotides moieties $[17,18]$. In aqueous environment, due to additional specific contributions of the polar heads to intermolecular interactions, i.e., $\pi$-stacking and hydrogen bonds, the presence of a bioinspired functional polar headgroup affects the self-assembly properties, and therefore this type of molecules had been extensively studied over the past years [19].

Molecular recognition at the air-water interface between nucleolipids and their complementary bases dissolved in the subphase, has 
been widely studied and focused upon adenine and uracil/thymine moieties in nucleolipids and also cytosine- and guanine-functionalized nucleolipids by surface experimental techniques [20-26]. On this framework, the present work presents an approach combining experimental, by surface pressure-area $(\pi-A)$ isotherms, Brewster angle microscopy (BAM) and polarization-modulation infrared spectroscopy infrared sprectroscopy (PM-IRRAS), techniques with molecular dynamics (MD) simulation techniques. This approach allows to describe in more detail the molecular recognition between cytosine and guanine molecules in an architecture prepared at the air-water interface by using a novel chemical system. Therefore, monolayers of cytosine-functionalized nucleolipid (CDP-DG) have been fabricated at the air-water interface where the aqueous subphase solves the complementary nitrogenous base at basic $\mathrm{pH}$. Under compression, the molecular organization of cytosine-based nucleolipid (CDP-DG) monolayer is influenced by guanine presence in the subphase. The formation of the complementary base paring in a monolayer is demonstrated and described by the combination of experimental and simulation techniques. The molecular organization implies a folding of the cytosine-based nucleolipid molecules in the monolayer during the guanine recognition. Furthermore, the molecular assembled is reproducible during several compression-expansion processes under set experimental conditions. Finally, mixed monolayers of CDP-DG and a phospholipid (DMPA) have been studied and a molecular segregation of DMPA molecules has been inferred by the additivity rule.

\section{Experimental section}

\subsection{Materials}

The nucleolipid 1,2-dipalmitoyl-sn-glycero-3-(cytidine diphosphate) (ammonium salt) powder ( $\geq 99 \%)$, CDP-DG, and phospholipid 1,2-dimyristoyl-sn-glycero-3-phosphate (sodium salt) ( $\geq 99 \%$ ), DMPA, were purchased from Avanti Polar Lipids and used as received. The purine bases, guanine hydrochloride (>99\%) and adenine $(98 \%)$ were obtained from Merck and used as well.

The basic subphase at $\mathrm{pH} 11$ was adjusted by $\mathrm{NaOH}(>99 \%)$ and supplied from Merck. The solutions for CDP-DG and DMPA were prepared in chloroform and $\mathrm{CHCl}_{3} / \mathrm{CH}_{3} \mathrm{OH} 3 / 1$, respectively, and used as spreading solvents. The pure solvents (Chromasol V® Plus) were purchased from Sigma Aldrich and used without further purification. Ultrapure water, produced by a Millipore Milli-Q unit, pre-treated by a Millipore reverse osmosis system $(>18.2 \mathrm{M} \Omega \mathrm{cm})$, was used as a subphase. The subphase temperature was kept at $21^{\circ} \mathrm{C}$.

\subsection{Methods}

Two different models of Nima troughs (Nima Technology, Coventry, England) were used in this work, both provided with a Wilhelmy type dynamometric system using a strip of filter paper: a NIMA $611 \mathrm{D}$ with one moving barrier for the measurement of surface pressure $(\pi)$-area $(A)$ isotherms and compression-expansion cycles, and a NIMA 601, equipped with two symmetrical barriers to record BAM images. The pure and mixed monolayers were compressed at a speed of $0.035-0.05 \mathrm{~nm}^{2} \cdot \mathrm{min}^{-1} \cdot \mathrm{molecule}^{-1}$.

Images of the film morphology were obtained by Brewster angle microscopy (BAM) with a I-Elli2000 (Accurion GmbH, Germany) using a Nd:YAG diode laser with wavelength $532 \mathrm{~nm}$ and $50 \mathrm{~mW}$, which can be recorded with a lateral resolution of $2 \mu \mathrm{m}$. The image processing procedure included a geometrical correction of the image, as well as a filtering operation to reduce interference fringes and noise. The microscope and the film balance were located on a table with vibration isolation (antivibration system MOD-2S, Accurion, Göttingen, Germany).

Monolayers of CDP-DG prepared on basic subphase at $\mathrm{pH} 11$ in presence and absence of guanine were transferred onto glass substrates by Langmuir-Schaefer (LS) technique, i.e., by horizontal touching method, at constant surface pressure $(\pi=27 \mathrm{mN} / \mathrm{m})$. Therefore, multilayers (10 layers) by sequential monolayer transfer were assembled. The transfer ratio was closed to unity for all transferences.

Polarization-modulation infrared reflection absorption spectroscopy (PM-IRRAS) was performed using a KSV PMI 550 instrument (KSV, Biolin Scientific Oy, Helsinki, Finland), and equipped with a highly sensitive MCT-detector and ZnSe photoelastic modulator. The Langmuir trough (KSV) was mounted so that the light beam reached the LS films at a fixed incidence angle of $76^{\circ}$. The incoming light is continuously modulated between $\mathrm{s}$ and $\mathrm{p}$ polarization at a high frequency. This allows the simultaneous measurement of spectra for the two polarizations, $s$ and $p$, thus the effect of water vapor is largely reduced. In such a way, the PM-IRRAS signal $(\mathrm{S})$ is the difference between $\mathrm{s}$ and $\mathrm{p}$ spectra, i.e. $\mathrm{S}=\Delta R / R=\left(R_{\mathrm{p}}-R_{\mathrm{s}}\right) /\left(R_{\mathrm{p}}+R_{\mathrm{S}}\right)$, where $R_{\mathrm{p}}$ and $R_{\mathrm{S}}$ are the parallel $\mathrm{p}$-and perpendicular s-polarized reflectances, respectively. The background spectrum of an uncoated glass substrate was recorded as the reference. In all the cases, the final spectrum was obtained by means of the accumulation of 600 scans. The spectra were recorded with $8 \mathrm{~cm}^{-1}$ resolution.

All the experiments were done in a large class 100 clean room at the temperature of $21^{\circ} \mathrm{C}$.

\subsection{Computer simulations}

Molecular mechanics (MM) and molecular dynamics (MD) calculations were performed by using Forcite with Dreiding force field. The charge was incorporated by using Charge equilibration (QEq). A cutoff of $1.85 \mathrm{~nm}$ was used to calculate the LJ interactions and short-range electrostatic interactions. For each MD simulation, the procedure was started with an optimized geometry followed by $20 \mathrm{ps}$ of NVT dynamics to heat the system to 298 K. Finally, after each MD simulation, the geometry was again optimized. This routine was repeated at least 3 times for each system.

3D Periodical conditions were considered as follows: two lipid leaflets confining a water layer and forming a sandwich structure in which the lipid heads point toward the water layer with different orientations. The absence of interactions between the two lipid leaflets along the $\mathrm{z}$ axis was achieved by introducing an empty gap of $2-4 \mathrm{~nm}$, as a function of surface area, above and below each lipid layers. The final dimensions of the computing box along $\mathrm{z}$ axes was $12.5 \mathrm{~nm}$.

\section{Results and discussion}

3.1. Surface pressure ( $\pi$ )-area (A) isotherms of CDP-DG nucleolipid at the air/water interface on different aqueous subphases

CDP-DG nucleolipid is a hybrid molecule composed of a nucleotide (Cytidine diphosphate) and a lipophilic moiety (dipalmitoyl derivative acid), see molecular structure in Scheme 1. Such structure is expected to be able for lateral recognition of the complementary base pairing, that is, guanine in the subphase [27].

Accordingly, its surface behaviour has been studied at the air/water interface by means of Langmuir technique. Therefore, CDP-DG films were prepared at the air-water interface and $\pi-A$ isotherms were registered on different aqueous subphases: pure water at $\mathrm{pH} 5.5$, basic subphase at $\mathrm{pH} 11$ both in absence and presence of guanine, and in presence of adenine. Such subphases has been set on basis of 


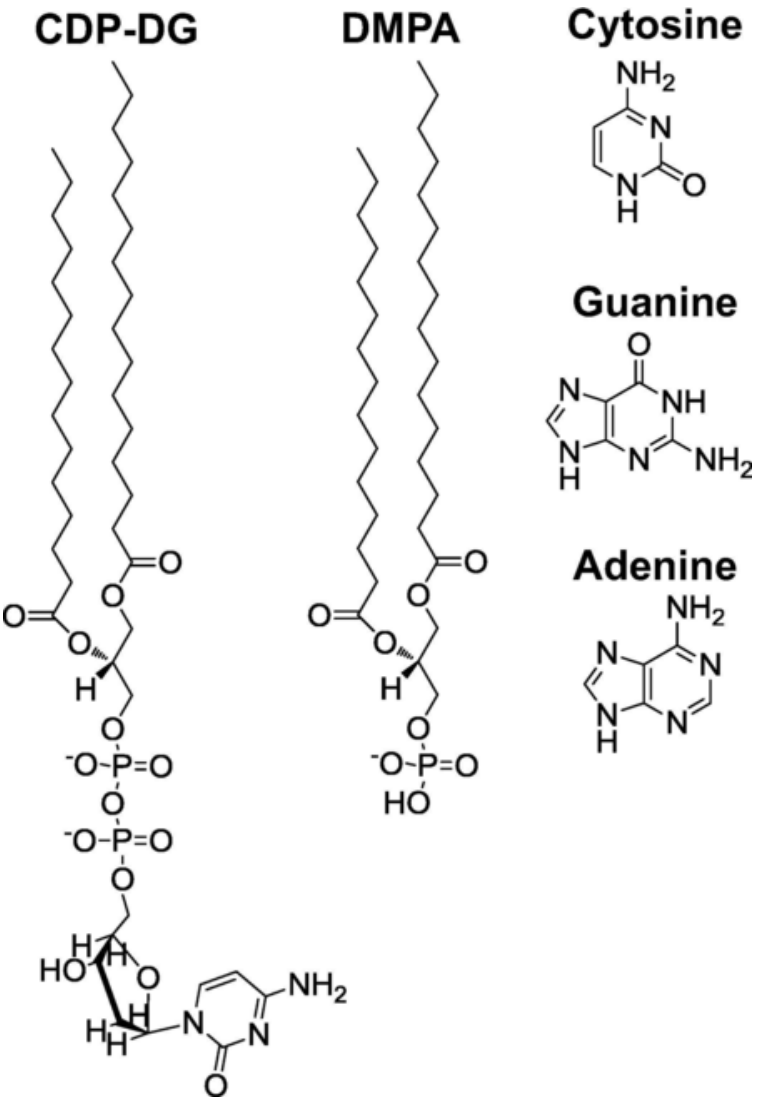

Scheme 1. Molecular Structures of 1,2-dipalmitoyl-sn-glycero-3-(cytidine diphosphate), CDP-DG, Dimyristoylphosphatidic Acid, DMPA, and purine and nitrogenous bases: Cytosine, Guanine and Adenine.

optimal solubility conditions for the complementary purine base, i.e., guanine.

Fig. 1 shows the $\pi-A$ isotherms under compression for a CDP-DG monolayer on water $\mathrm{pH} 5.5$ (black line), basic subphase $\mathrm{pH} 11$ in absence of guanine (red line) and basic subphase $\mathrm{pH} 11$ containing guanine $10^{-4} \mathrm{M}$ (green line).

As can be seen, in absence of purine base, an increase of subphase $\mathrm{pH}$ leads to two phenomena: (1) an expansion of the molecular area

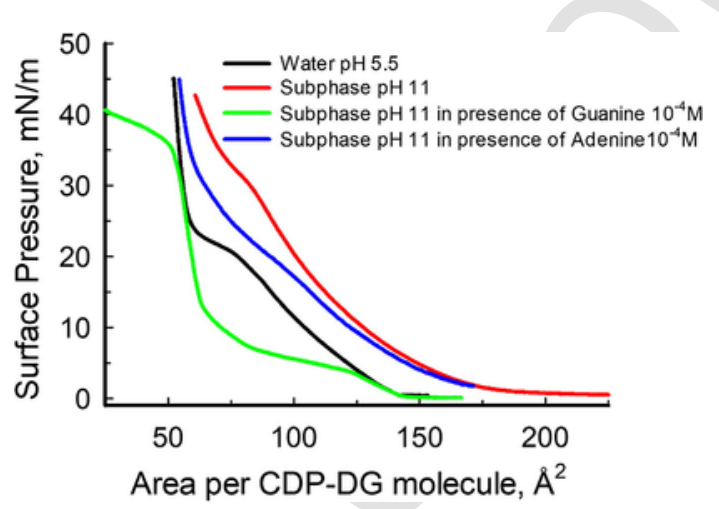

Fig 1. $\pi-A$ isotherms of a CDP-DG monolayer prepared at air/water interface on different subphases: water (black line) and basic subphase $\mathrm{pH} 11$ in absence (red line) and presence of purine bases, Guanine (green line) and Adenine (blue line), respectively. (For interpretation of the references to colour in this figure legend, the reader is referred to the web version of this article.) and (2) a shift of the phase transition from $75 \AA^{2} /$ molecule and $20 \mathrm{mN} / \mathrm{m}$ for the film on pure water at neutral $\mathrm{pH}$, upto around $85 \AA^{2} / \mathrm{molecule}$ and $30 \mathrm{mN} / \mathrm{m}$ on basic subphase at $\mathrm{pH} 11$. Moreover, the presence of the guanine in the subphase (Fig. 1, green line) produces outstanding effects on the CDP-DG matrix as show the registered isotherms: (i) the $\pi-A$ isotherm converges to that obtained on water $\mathrm{pH} 5.5$ at very low $(\pi<5 \mathrm{mN} / \mathrm{m})$ and high surface pressures $(25<\pi<35 \mathrm{mN} / \mathrm{m})$

(ii) two phase transitions are clearly distinguished, at low surface pressures $(5<\pi<10 \mathrm{mN} / \mathrm{m})$ and high surface pressures $(\pi>35 \mathrm{mN} / \mathrm{m})$.

Furthermore, as molecular recognition control, the $\pi-A$ isotherm of the CDP-DG monolayer on aqueous subphase containing $10^{-4} \mathrm{M}$ adenine was run. The $\pi-A$ isotherm (Fig. 1, blue line) show convergence at low and high surface pressures with the isotherm obtained in absence of nitrogenous base (Fig. 1, red line) and any phase transitions. These results are indicative of any interaction between cytosine group of nucleolipid and adenine, as expected.

By comparison such $\pi-A$ isotherms obtained on water subphase in absence of guanine (Fig. 1, black line) and that on $\mathrm{pH} 11$ and $10^{-4} \mathrm{M}$ guanine (Fig. 1, green line), the phase transition registered at around $5 \mathrm{mN} / \mathrm{m}$ could be related to that observed on water, although is shifted to high surface area and low surface pressure. However, the phase transition at high surface pressures can be related to a molecular recognition between the cytosine group of the nucleolipid monolayer and the guanine molecules in the basic subphase.

Simultaneously to the $\pi-A$ isotherms during the compression process, the morphological structure of the CDP-DG film at different subphases, has been directly visualized by BAM. The BAM images are shown in Fig. 2.

Fig. 2 shows the morphological features of the CDP-CG monolayer under compression on different aqueous subphases: the upper row shows the behaviour on pure water, the middle one corresponds to basic subphase $\mathrm{pH} 11$, and the lower one to the presence of $10^{-4} \mathrm{M}$ guanine.

On pure water (Fig. 2A), and at low surface pressure a gas phase is clearly observed and under compression the CDP-DG molecules form small circular domains that fused at high surface pressure. However, when the subphase $\mathrm{pH}$ increases (Fig. 2B), some small bright spots are detected at very large molecular areas and under compression irregular domains are formed until covering all the focused area. A behaviour in between those above is observed when guanine is present in the basic subphase (Fig. 2C), that is, gas phase at low surface pressure, formation of small irregular domains during the first phase transition and covering all the focus area with a monolayer where many bright spots emerge during the second phase transition.

BAM images, as described previously from the $\pi-A$ isotherm results, also show the different behaviour of the CDP-DG monolayer as a function of the subphase $\mathrm{pH}$ and notoriously different when guanine molecules are in the basic subphase. Details of the morphological characteristics of the CDP-DG film on basic subphase in presence of guanine during the compression process upto the collapse are given in SI1 (Supporting Information, SI).

As discussed above, the second phase transition registered at the $\pi-A$ isotherm of the nucleolipid matrix on basic subphase containing guanine $10^{-4} \mathrm{M}$ can be due to the specific interaction between guanine and cytosine, that occurs at high surface pressure. Therefore, to infer on this phenomenon, two compression-expansion cycles of a CDP-DG monolayer reaching the collapse at approximately $30 \mathrm{mN} / \mathrm{m}$ and $50 \AA^{2} /$ molecule, were performed. The results are shown in Fig. 3.

Fig. 3 shows a great hysteresis between 1 st and 2 nd compression cycles when the collapse surface pressure is reached during the first compression process, and a significant shrinking of the isotherm dur- 


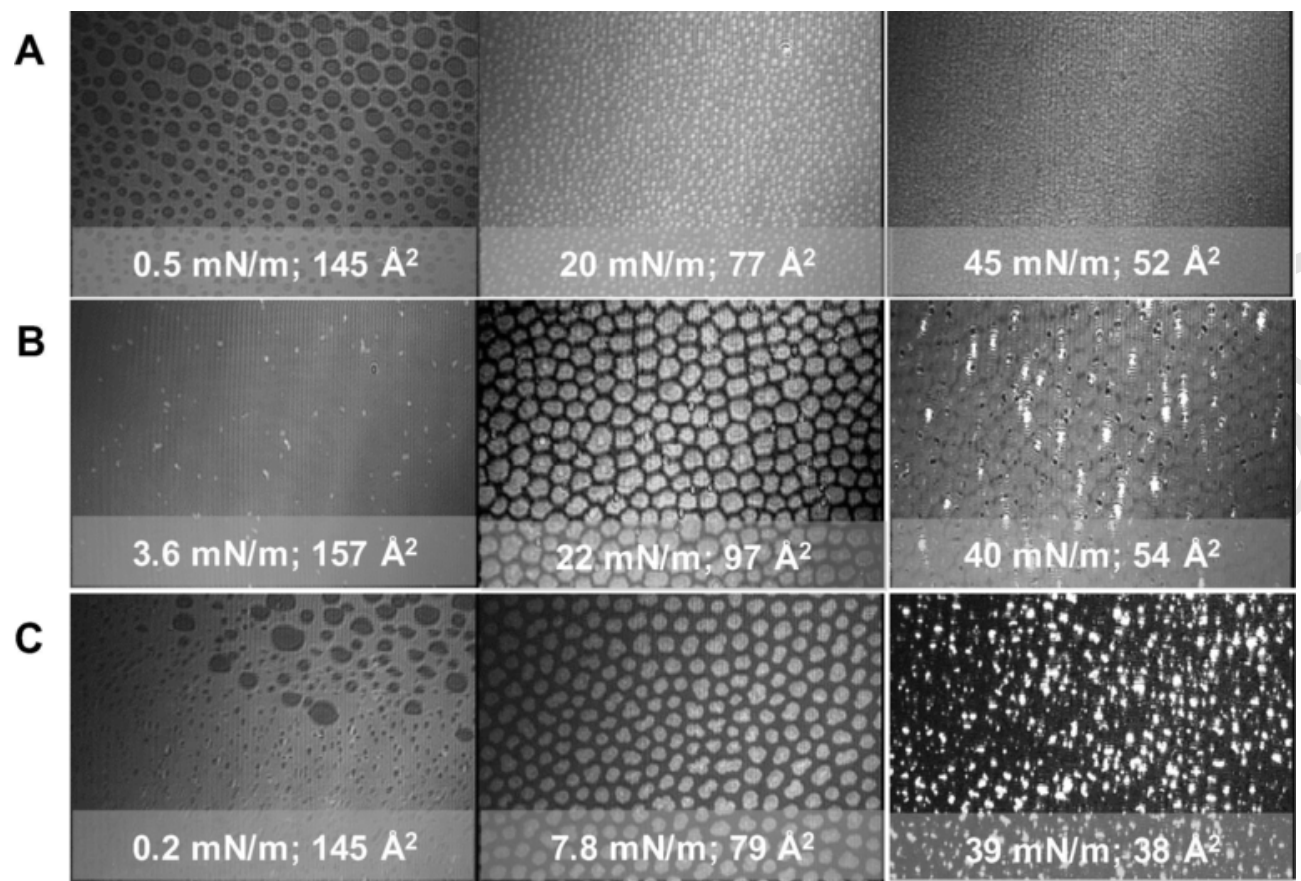

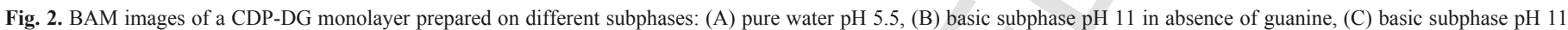
containing Guanine $10^{-4} \mathrm{M}$. The molecular areas indicated in the images are expressed per CDP-DG molecule. Image width: $430 \mu \mathrm{m}$.

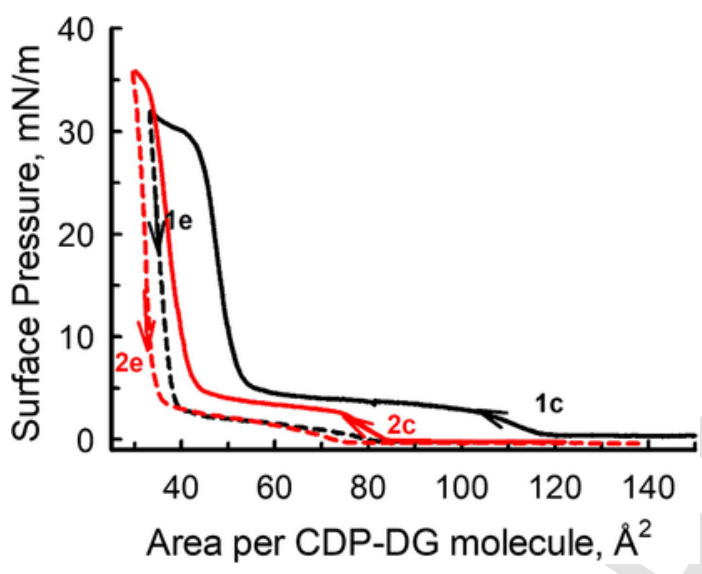

Fig. 3. Compression-Expansion cycles of a CDP-DG monolayer on a basic subphase $\mathrm{pH} 11$ in presence of Guanine $10^{-4} \mathrm{M}$ : 1 st compression-expansion $(1 \mathrm{c}-\mathrm{e})$, black lines 2nd compression-expansion ( $2 \mathrm{c}-\mathrm{e})$, red lines. (For interpretation of the references to colour in this figure legend, the reader is referred to the web version of this article.)

ing the second compression cycle is registered. This phenomenon may be ascribed to a conformational change of CDP-DG molecules arriving to the collapse surface pressure and in presence of guanine, complementary nitrogenous base of the cytosine polar group of the nucleolipid: before the collapse, the polar group of the nucleolipid molecules are oriented properly to bind the guanine molecules in basic subphase and extending their surface area

by contrary, after the collapse the polar groups of the CDP-DG molecules redirect ejecting the guanine molecules [28].

Therefore, to confirm the hypothesis above described, several bounded compression-expansion cycles have been made, and a monolayer of CDP-DG on a basic subphase $\mathrm{pH} 11$ containing guanine $10^{-4} \mathrm{M}$ has been compressed and expanded during three cycles upto $27 \mathrm{mN} / \mathrm{m}$. The results are shown in Fig. 4. No hysteresis is registered. Thus, it can be confirmed that before the collapse, the orienta-

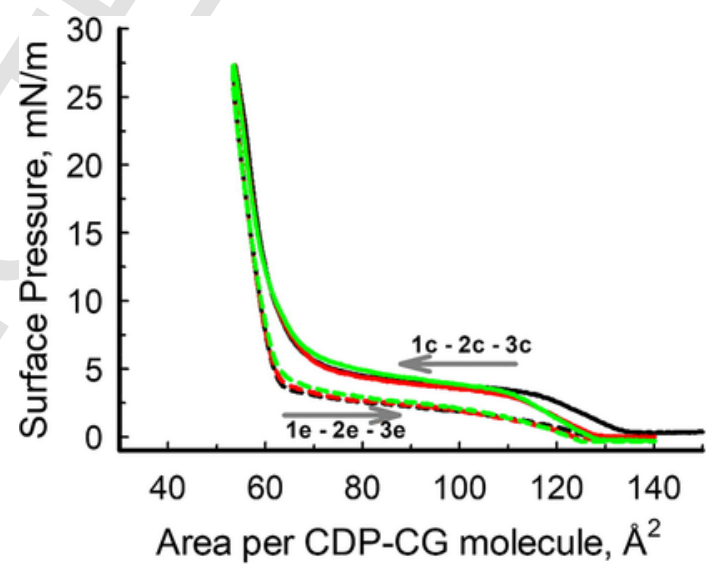

Fig. 4. Bounded expansion-compression cycles of CDP-DG on basic solution pH 11 containing Guanine $10^{-4} \mathrm{M}$.

tion of the polar group is directed to bind guanine molecules in the basic subphase.

Details of the morphological characteristics of the CDP-DG film on basic subphase in presence of guanine during the compression process upto the surface pressure before collapse, are given in SI2. No differences between the bounded cycles were noticed.

\subsection{PM-IRRAS of CDP-DG nucleolipid transferred by horizontal touching from the air/water interface to glass support}

PM-IRRAS has been widely used for studying of monolayers formed by phospholipid molecules at the air-water interface [29-36] and the assignment of the corresponding bands to the different chemical groups has been described in detail, as well.

The binding of the guanine and polar group of the nucleolipid molecules is mainly due to the hydrogen bonds as expected for gua- 
nine-cytosine interaction. However, under basic conditions, subphase $\mathrm{pH} 11$, which are necessary for the dissolution of guanine as absorbate, an intense negative band between 1800 and $1600 \mathrm{~cm}^{-1}$ in the PM-IRRAS spectra, also recorded on clean subphase (background) gives rise and similar to that described elsewhere [37]. This artefact makes impossible to analyze the spectral region of greatest interest at the air-water interface. Nevertheless, the existence of interactions between the nucleolipid monolayer and the guanine dissolved in the subphase can be proved on LS films once the modified substrate is rinsing with pure water to remove the large bands corresponding to water/ $\mathrm{NaOH}$ absorption in the low wavenumber region as showed in SI3.

To confirm the binding of the guanine and polar group of the nucleolipid molecules is mainly due to the hydrogen bonds as expected for guanine-cytosine interaction, the CDG-DG film prepared on basic subphase $\mathrm{pH} 11$ was transferred at constant surface pressure ca. $27 \mathrm{mN} / \mathrm{m}$ on glass support by Langmuir-Schaefer method, and PM-IRRAS spectra were measured.

Fig. 5 shows PM-IRRAS spectra corresponding to $10 \mathrm{LS}$ layers of CDP-DG transferred from aqueous interface at $\mathrm{pH} 11$ in absence (blue line) and presence (red line) of guanine, on the modified substrate has been rinsed with ultrapure water and dried under air flow, two times. Also, the selected PM-IRRAS signals of such multilayers and their assignments are given in Table 1.

At high wavenumbers two characteristic signals of lipids with bands originated from $\mathrm{C}-\mathrm{H}$ stretches at 2917 and $2848 \mathrm{~cm}^{-1}$ and as-

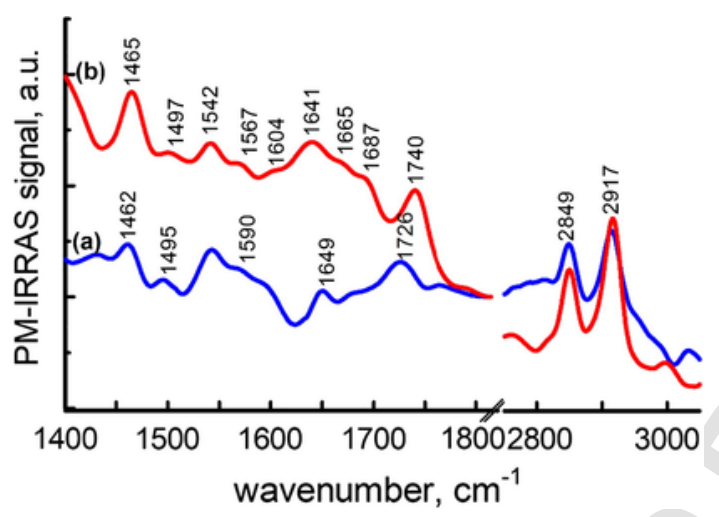

Fig. 5. PM-IRRAS spectra for $10 \mathrm{LS}$ multilayers of CDP-DG film prepared at air-water interface at surface pressure ca. $27 \mathrm{mN} / \mathrm{m}$ and aqueous subphase $\mathrm{pH}$ 11: (a) in absence and (b) in presence of guanine $10^{-4} \mathrm{M}$, blue and red lines, respectively. (For interpretation of the references to colour in this figure legend, the reader is referred to the web version of this article.)

Table 1

The major characteristic peaks of CDP-DG and CDP-DG/Guanine LS films and their possible assignments ${ }^{\mathrm{a}, \mathrm{b}}$.

\begin{tabular}{lll}
\hline assignment & CDP-DG & CDP-DG/Guanine \\
\hline$\nu_{\mathrm{a}}\left(\mathrm{CH}_{2}\right)$, asym stretch & 2917 & 2917 \\
$\nu_{\mathrm{s}}\left(\mathrm{CH}_{2}\right)$, sym stretch & 2848 & 2850 \\
$\nu(\mathrm{C}=\mathrm{O})$, ester stretch & 1726 & 1740 \\
$\nu\left(\mathrm{C}=\mathrm{O}^{\text {cytosine }}\right)$, stretch & 1687 & 1689 \\
$\nu\left(\mathrm{C}=\mathrm{O}^{\text {guanine }}\right)$, stretch & & 1668 \\
$\delta\left(\mathrm{NH}_{2}\right)$, scissor mode & 1649 & 1641 \\
$\nu(\mathrm{C}=\mathrm{N})+\nu(\mathrm{C}=\mathrm{C})$, ring stretch & 1590 & 1605 \\
ring stretch & $1567,1542,1495$ & $1567,1542,1497$ \\
$\delta\left(\mathrm{CH}_{2}\right)$, scissor mode & 1462 & 1465 \\
\hline
\end{tabular}

${ }^{a}$ Band positions are given in $\mathrm{cm}^{-1}$.

${ }^{\text {b }}$ Reference Wang et al. [38] signed to the antisymmetric and symmetric $\mathrm{CH}_{2}$ stretching modes $\left[\nu_{\mathrm{a}}\left(\mathrm{CH}_{2}\right)\right.$ and $\left.\nu_{\mathrm{s}}\left(\mathrm{CH}_{2}\right)\right]$ of hydrocarbon chains, respectively, are detected for both films. These wavenumbers are characteristic of preferential all-trans conformers in highly ordered alkyl chains.

As previously described [39-45], the intensity of the tension vibrations of the methylene group depends on the orientation of the alkyl chains with respect to the glass substrate. However, as prepared LS CDP-DG films in the absence and presence of guanine those bands shown different height (see Fig. 5) and this fact should be mainly attributed to surface concentration rather than to orientation molecular variations (see Figure SI4). Accordingly to Fig. 1, at about $27 \mathrm{mN} / \mathrm{m}$, the CDP-DG monolayer prepared at air/water interface on basic subphase $\mathrm{pH} 11$ in absence (red line) has a larger area than that in presence of Guanine (green line), about 89 vs. $57 \mathrm{~A}^{2}$. Therefore, and taking into account that the transfer ratio of the LS films is practically one, the surface concentration can be corrected by expressing the PM-IRRAS signal in a normalized manner, that is, multiplying the corresponding spectrum by its registered molecular area under surface pressure. Figure SI4 shows the normalized delta/sigma signal of the bands $\nu \mathrm{a}\left(\mathrm{CH}_{2}\right)$ and $\nu \mathrm{s}\left(\mathrm{CH}_{2}\right)$ and as can be seen for both LS films the spectra practically coincide.

At the region of low wavenumbers, a slightly different behaviour between those films is observed. Therefore, the vibrational frequency at $1726 \mathrm{~cm}^{-1}$ is assigned to the $\mathrm{C}=\mathrm{O}$ stretching vibration $[\nu(\mathrm{C}=\mathrm{O})]$, from the ester group and the band at $1462 \mathrm{~cm}^{-1}$ is caused by the scissor stretch of alkyl chains $\left[\delta\left(\mathrm{CH}_{2}\right)\right]$. At the region $1490-1690 \mathrm{~cm}^{-1}$, the small bans are related to the cytosine group stretching. Particularly, the signals at 1495,1542 and $1567 \mathrm{~cm}^{-1}$ are arised from ring stretching vibration. Additionally, the shoulder at $1590 \mathrm{~cm}^{-1}$ is due to the coupled stretching vibration of $\mathrm{C}=\mathrm{N}$ and $\mathrm{C}=\mathrm{C}$ bonds in the cytosine moieties. Furthermore, the broad band detected at $1649 \mathrm{~cm}^{-1}$ can be mainly assigned to $\mathrm{NH}_{2}$ scissoring vibration, while the shoulder at $1687 \mathrm{~cm}^{-1}$ corresponds to the stretch mode of $\mathrm{C}=\mathrm{O}$ group belong to the cytosine $\left[\nu\left(\mathrm{C}=\mathrm{O}^{\text {cytosine }}\right)\right]$. Nonetheless, both bands can be also governed by the hydrogen interactions between neighbour molecules $\left[\mathrm{C}=\mathrm{O}^{\text {cytosine }} \ldots \mathrm{H}-\mathrm{N}^{\text {cytosine }}-\mathrm{H}\right]$.

By contrary, in the case of the CDP-DG multilayers fabricated from Langmuir monolayer of nucleotide spread onto basic subphase containing guanine (red line, Fig. 5), the band at $1590 \mathrm{~cm}^{-1}$ vanishes and a new one at $1605 \mathrm{~cm}^{-1}$ emerges. This band can be related to the $\mathrm{C}=\mathrm{N}$ stretching vibration links together $\mathrm{C}=\mathrm{C}$ stretching modes from each cytosine and guanine rings. These different features for the two different multilayers suggest a reorganization and/or orientation change of the cytosine ring group in the nucleolipid molecule probably due to the formation of hydrogen bonds with the guanine molecules in the subphase $\left[\mathrm{C}=\mathrm{N}^{\text {cytosine }} \ldots \mathrm{H}-\mathrm{N}^{\text {guanine }}\right]$. This binding is confirmed by the shift of the band at $1649 \mathrm{~cm}^{-1}-1641 \mathrm{~cm}^{-1}$ that notes the molecular recognition between guanine and cytosine by $\mathrm{H}-\mathrm{N}^{\text {guanine }}-$ $\mathrm{H} . . \mathrm{O}=\mathrm{C}^{\text {cytosine }}$, and the appearance of a new band at $1668 \mathrm{~cm}^{-1}$ assigned to $\nu\left(\mathrm{C}=\mathrm{O}^{\text {guanine }}\right)$ and coming from $\mathrm{O}=\mathrm{C}^{\text {guanine }} \ldots \mathrm{H}-\mathrm{N}^{\text {cytosine }}$ $-\mathrm{H}$ interaction.

Finally, it is noteworthy the shift of the $\mathrm{C}=\mathrm{O}$ (ester group of the alkyl chains) stretching frequency happening from $1726 \mathrm{~cm}^{-1}$ to $1740 \mathrm{~cm}^{-1}$ when guanine molecules are in the aqueous subphase during the LS process, and corresponding to a dry ether group.

These results not only reproduce those published elsewhere $[38,46]$, but additionally evidence the strong interaction between the ring groups of the cytosine and guanine par that leads to a framework where the orientation of the molecules in the monolayer avoids the hydration of the $\mathrm{C}=\mathrm{O}$ groups during the horizontal transfer process. 


\subsection{Computer simulations}

To predict structures and dynamics between biological adsorbed molecules and a main-group of inorganic molecules, Dreiding has been showed as a very suitable tool $[47,48]$. Thus, molecular mechanics calculations using the Dreiding force field were applied to self-assembled monolayer configurations of the nucleic acid base adenine adsorbed on graphite surfaces [49]. Also, Dreiding force field has been used for investigating a wide variety of DNA sequences and sizes containing coding and non-coding, random and real, homogeneous or heterogeneous sequences in the range from 2 to 40 base pairs. Dreiding force field has been shown to improve the accuracy that the geometry of the hydrogen system adopts by using the 10-12 terms and includes the angle between the hydrogen donor $(\mathrm{D})$, the hydrogen $(\mathrm{H})$, and the hydrogen acceptor (A) [50]. Additionally, a series of porphyrinogens related to coproporphyrinogen-III have been modeled by molecular dynamics simulations (Dreiding force field). The results demonstrated that the porphyrinogen framework is highly flexible and can take on a number of conformations. Hydrogen bonding interactions to propionic acid side chains have a significant influence on the preferred conformations of these species, although this is overwhelmed by interactions with water molecules for solvated porphyrinogen [51].

On the basis of the potential suitability of Dreiding above described, here it has been applied for the simulation of DNA base-pairing.

Each box was built incorporating 4 nucleolipid (CDP-DG) units per leaflets. The dimensions along the $\mathrm{x}$ - and $\mathrm{y}$-axis depend on simu-

Table 2

$\mathrm{XYZ}$ dimensions values of the computational box.

\begin{tabular}{lllll}
\hline & $\mathrm{x}$-axis nm & y-axis $\mathrm{nm}$ & $\pi \mathrm{mN} / \mathrm{m}$ & $A_{\mathrm{NL}} \mathrm{nm}^{2}$ \\
\hline \multirow{2}{*}{ Absence } & 3.0 & 2.2 & 5 & 1.65 \\
& 2.14 & 1.53 & 30 & 0.82 \\
Presence & 2.42 & 1.82 & 5 & 1.10 \\
& 1.8 & 1.12 & 30 & 0.5 \\
\hline
\end{tabular}

lated surface pressures ( 5 and $30 \mathrm{mN} / \mathrm{m}$ ). The dimensions obtained both in presence and absence of guanine are shown in Table 2.

Snapshots of the system at low surface pressure have been depicted in SI4: in absence (SI5A) and in presence (SI5B) of guanine.

As above described, the nucleolipid isotherms at the air-water interface, in the absence or presence of the guanine at the aqueous subphase, show significant differences. Briefly, those differences due to the presence of guanine are fundamentally three: (1) large reduction of the surface area per nucleolipid molecule;(2) decrease of the surface pressure at which the phase transition occurs, that is, $30 \mathrm{mN} / \mathrm{m}$ in the absence whilst $5 \mathrm{mN} / \mathrm{m}$ in the presence of guanine

and (3) drop of the collapse surface pressure.

The MD simulations show that at low surface pressure $(5 \mathrm{mN} / \mathrm{m})$, and in absence of guanine, the cytosine group is folded, so that its plane is located almost parallel to the interface. However, in presence of guanine, the polar group of the nucleolipid molecules at the matrix in the interface modify its conformation extending into the aqueous subphase, as a result of the formation of hydrogen bonds between cytosine and guanine. This phenomenon explains accordingly the contraction of the nucleolipid isotherm in Guanine presence with respect to that measured in absence of the purine base, see Fig. 1.

Also, at high surface pressure, a surface area reduction is appreciated. Thus, at $30 \mathrm{mN} / \mathrm{m}$, the surface area per CDP-DG molecule in guanine absence is $\sim 0.8 \mathrm{~nm}^{2}$, while in guanine presence is approx. $0.5 \mathrm{~nm}^{2}$. The MD simulation indicates that the nucleolipid undergoes a conformational change as a consequence of the formation of hydrogen bonds between cytosine and guanine, see Fig. 6. However, such shrinkage of the surface area cannot be explained because exclusively on the conformation change: strong repulsion between negatively charged phosphate groups of the NL units can be expected. The analysis of the results obtained by MD simulation shows that the units of guanine and cytosine are separately stacked at distances of the order of $0.33 \mathrm{~nm}$, see Fig. 6 . Thus, the stacking occurs not only between guanidine groups, but also between the cytosine groups belonging to the polar group of the nucleolipid molecules. The pi-stacking (also called $\pi-\pi$ stacking) is a noncovalent attractive interaction between aromatic rings. This stacking not only stabilizes the (c)

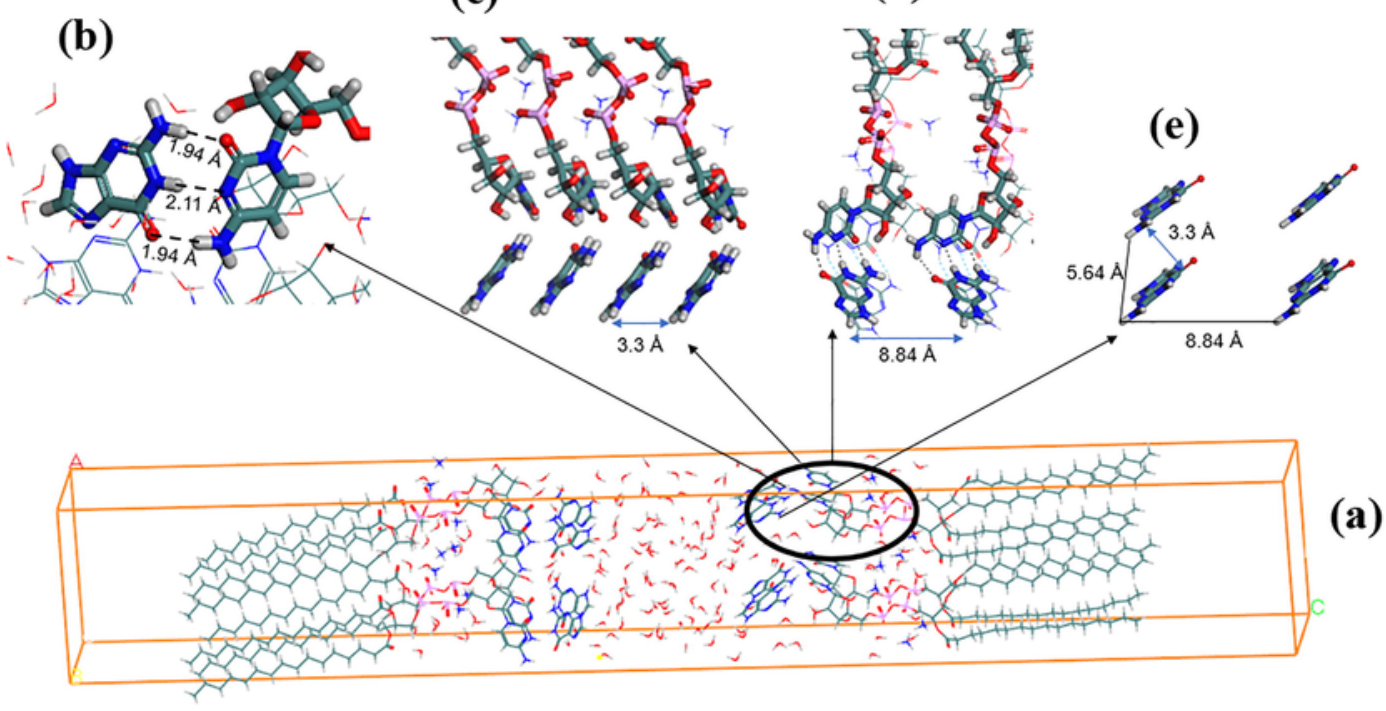

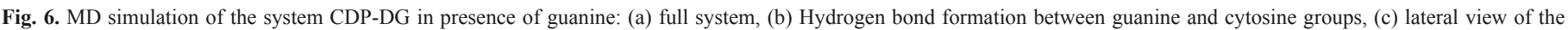
periodic system, (d) frontal view of the periodic system, (e) view of the molecular organization of a system formed only by guanine molecules. 
monolayer, but also favors its contraction and partially compensates the repulsion energy between phosphate groups.

Furthermore, this strong stacking should be the reason for the decrease of the surface pressure at which the phase transition occurs when guanine is present in the aqueous subphase $(\sim 5 \mathrm{mN} / \mathrm{m})$, compared to that registered in its absence $(\sim 30 \mathrm{mN} / \mathrm{m})$.

As discussed above, the third effect of the guanine presence on the CDP-DG isotherm, see Fig. 1, is the drop of the collapse pressure. In previous works $[52,53]$, it has been observed that certain well-organized monolayers may collapse toward trilayers where first and third monolayers organize the polar headgroups pointing to the aqueous subphase, whereas the intermediate layer displays the polar headgroups pointing to the air. In such a way, the polar groups of the monolayers further away from the water must be in contact. Those type of trilayers are stabilized by the strong attractive interactions between the head groups in contact [53]. Therefore, in the present work as previously described, the collapse of the CDP-DG isotherm may be favored by attractive interactions like stacking-type interactions between the guanine molecules of the alternating layers, see Fig. 6.

\subsection{Dilution of CDP-DG monolayer by fabrication of mixed CDP- $D G: D M P A$ films at the air-water interface}

The combination of nucleic acid derivatives with lipids renders hybrid materials with interesting applications and can confers properties of interest to the system, like biocompatibility, biodegradability, etc. Also, to make easy the handling with synthetic amphiphilic nucleolipids, a dilution of the cytosine-based nucleolipid monolayer with a lipid is considered. Therefore, to organize the CDP-DG molecules in a lipid environment approaching to a membrane arrangement, the nucleolipid was mixed with DMPA phospholipid.

Previously, as reference $\pi-A$ isotherms of DMPA Langmuir films prepared on different subphases: on pure water, basic subphase in absence and presence og guanine, and basic subphase with adenine (SI6). The results note differences between netral or basic subphases, an expansion of the isotherms at low surface pressure upto reaching the phase transition at ca. $35 \mathrm{mN} / \mathrm{m}$ and shrinkage after the phase transition. Further, such effect is not related to the presence of the nitrogenous bases in the basic subhases, it seems to be related only with the increases of $\mathrm{pH}$.

Simultaneously to the isotherms, BAM images were taken during the compression process (SI7). The morphological features of DMPA monolayer on the different basic subphases $\mathrm{pH} 11$ in absence and presence of guanine $10^{-4} \mathrm{M}$ are clearly different of the corresponding for DMPA on pure water [54]. The images show a the effect on the domain formation under compression of the monolayer: in presence of guanine, the domains significantly reduce their size to form small dots and the anisotropy detected in the leave like domains typical of DMPA on water subphase disappears.

Following, mixed DMPA:CDP-DG films were prepared en different molar ratio $(1: 0$

$5: 1$

$2: 1$

$1: 1$

$1: 2$

$0: 1)$. As an example, Fig. 7 shows the variation of $\pi-A$ isotherms due to the dilution effect of DMPA molecules in the mixed DMPA:CDP-DG 1:1 monolayer fabricated at air/water interface with basic subphases in absence of purine derivatives (black line), as reference, and in presence of Guanine $10^{-4} \mathrm{M}$ (red line) and Adenine $10^{-4} \mathrm{M}$ (blue line). Furthermore, the selectivity interaction between nitrogeous pair bases in the mixed monolayer was also explored. The

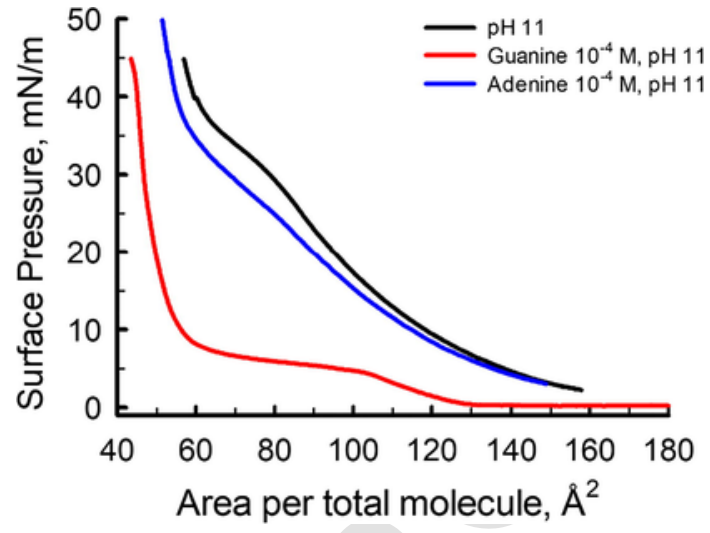

Fig. 7. $\pi-A$ isotherms of a mixed DMPA:CDP-DG 1:1 monolayer fabricated at air/water interface with basic subphases in absence of purine derivatives (black line), as reference, and in presence of Guanine $10^{-4} \mathrm{M}$ (red line) and Adenine $10^{-4} \mathrm{M}$ (blue line). (For interpretation of the references to colour in this figure legend, the reader is referred to the web version of this article.)

results clearly demonstrate the specificity between nucleic bases in spite of the dilution of the nucleolipid monolayer with phospholipid molecules. Details of the $\pi-A$ isotherms recorded on basic subphases in absence and presence of guanine $10^{-4} \mathrm{M}$, as well as the morphological characteristics are shown in SI8-SI10. The $\pi-A$ isotherms were repeated at least three times and good reproducibilitly was obtained. From the isotherms show in SI8-SI10 can note that the dilution of CDP-DG with DMPA is better when the nucleolipid concentration is higher.

An understanding of the interaction between CDP-DG and DMPA is provided by means of a miscibility study. The nature of the miscibility can be investigated from the variation of mean molecular area, $A_{\mathrm{m}}$, versus surface pressure, $A_{\mathrm{m}}(\pi)=X_{1} A_{1}(\pi)+X_{2} A_{2}(\mathrm{p})$, Eq. (1), where $A_{\mathrm{i}}$ is the molecular area of the pure component $\mathrm{i}$ film at the relevant surface pressure and $X_{\mathrm{i}}$ is the molar fraction of the component $\mathrm{i}$ in the mixed film.

Coincident areas would correspond to a situation in which a pure DMPA monolayer coexists with the equimolecular mixed film at the air-water interface. Obviously, deviations from the additivity rule in the isotherms suggest that the molecules are differently arranged along that pressures range. Deviations from the additivity rule are expected as a result of special molecular organization characteristics such as filling of the empty space by one component or changes in molecular packing as a result of interlocking of the different components. The size of the deviation, the form of the curves, and the mole fraction for which the maximum deviation occurs will depend strongly on the amphiphilic nature of the two components in the mixed layer [55-57].

The comparison between the experimental molecular areas (symbols) with the mean molecular areas, calculated by Eq. (1), at different surface pressure is shown in Fig. 8, in which it can be seen clearly that the mixed monolayers, in absence of guanine, do not follow the additivity rule. Miscibility results show a positive deviation behaviour over the ideality in basic subphase. The experimental molecular areas in all cases are larger than the corresponding calculated isotherm. Furthermore, the positive deviation from the additivity rule depends on the molar fraction of the CDP-DG in the mixture, becoming larger as the density of cytosine-based nucleolipid in the mixed film decreases.

As the mixed film is compressed, the distance between molecules in the monolayer decreases and their hydrophobic interactions increases. Consequently, the mixture of CDP-DG and DMPA exhibits a 


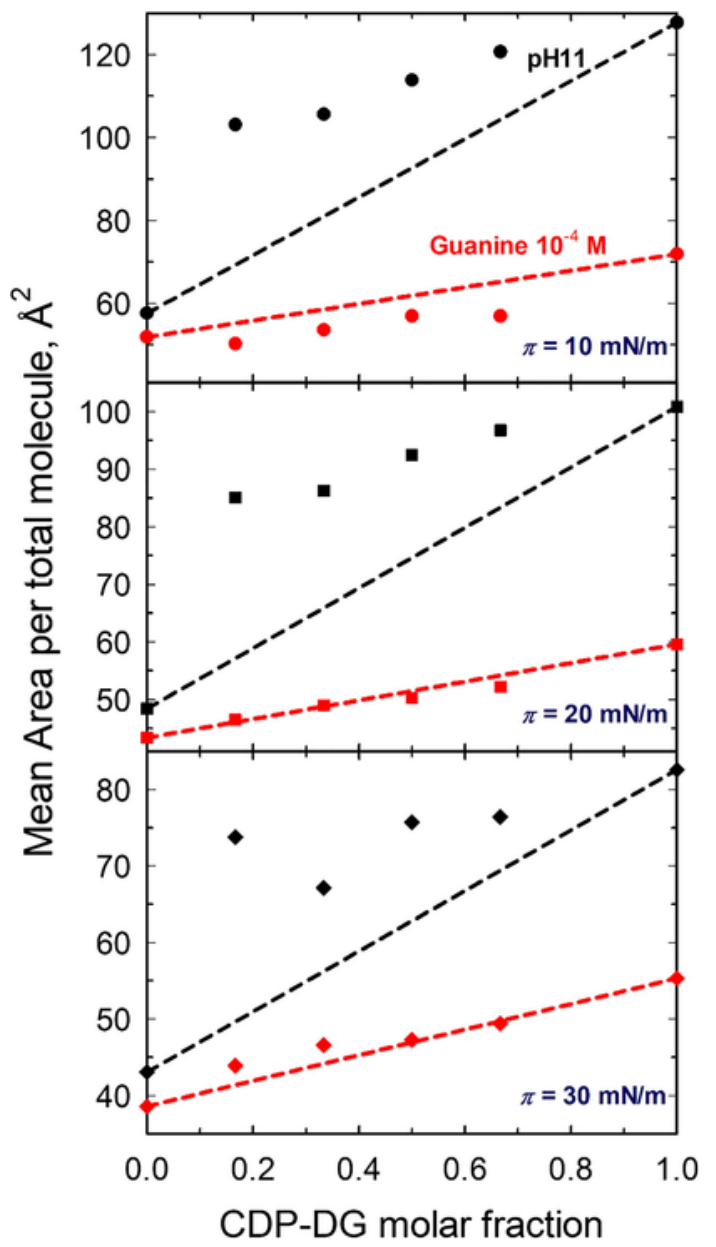

Fig. 8. Plots of area per molecule and deviations of ideal behaviour (lines) of a mixed DMPA:CDG-DP 1:1 film formed on basic subphase $\mathrm{pH} 11$ in presence (red) and absence (black) of Guanine at 10 (circles), 20 (squares) and $30 \mathrm{mN} / \mathrm{m}$ (diamonds). (For interpretation of the references to colour in this figure legend, the reader is referred to the web version of this article.)

positive deviation from the additivity rule. When the mixed film is compressed further, those interactions become so strong that the amphiphilic molecules become less complatible and different regions can be formed, as detected in BAM images (SI10a).

Surprisingly, such phenomenon does not occurr when guanine is present in the basic subphase: as a funtion of the surface pressure, the deviation of the mixed monolayer with respect to the additivity rule is slightly negative at low surface pressure, almost coincident at intermediate surface pressures, and slightly positive at high $\pi$. This behaviour is in good agreement with the BAM images shown in SI10b. With Guanine the molecular organization of the mixed film is because a hypothetical situation of two coexisting immiscible monolayers.

\section{Conclusions}

In this work, monolayers of a hybrid cytosine-based nucleolipid, CDP-DG, at basic subphase have been prepared at the air-water interface both in absence and presence of guanine by mechanical stimulus. The $\pi-A$ isotherms and successive compression-expansion cycles highlight different organization of the CDP-DG in presence of guanine as a function of the surface pressure during the mechanical stimulus, that is, below or above phase transition. Differences were also directly observed by BAM. Furthermore, PM-IRRAS spectra of Langmuir-Schaefer CDP-DG monolayers suggest a reorganization and/or orientation change of the cytosine ring group in the nucleolipid molecule probably due to the formation of hydrogen bonds with the guanine molecules in the subphase. These results also evidence the strong interaction between the ring groups of the cytosine and guanine par. Finally, computer simulations show how as a function of the surface pressure, in absence of guanine, the cytosine group is folded, so that its plane is located almost parallel to the interface. However, in presence of guanine, the polar group of the nucleolipid molecules at the matrix in the interface modify its conformation extending into the aqueous subphase, because of the formation of hydrogen bonds between cytosine and guanine.

The present work presents an approach combining experimental with molecular dynamics (MD) simulation techniques, that with respect to previous research, this approach allows to describe in more detail the molecular recognition between cytosine and guanine molecules in an architecture prepared at the air-water interface by using a novel chemical system.

Just like that, by combining surface experimental techniques, i.e., surface pressure $(\pi)$-area $(A)$ isotherms, Brewster angle microscopy (BAM), infrared spectroscopy (PM-IRRAS) with computer simulations, not only the formation of the complementary base pairing $(\mathrm{Cy}$ tosine-Guanine) established mainly by hydrogen bonds, is demonstrated, but also is provided the folding of the cytosine-based nucleolipid molecules in the monolayer is forced to change its orientation during the compression process and the guanine recognition in the basic subphase. Moreover, that change orientation is reversible during several compression-expansion processes under set experimental conditions.

Furthermore, the specific interaction between nitrogenous bases has been also demonstrated along the study of the CDP-DG monolayer fabricated at the air-water interface. Finally, the dilution of the cytosine-based nucleolipid monolayer with DMPA has resulted in mixed monolayers where a slight segregation between two molecules has been inferred by the additivity rule.

From what is reported, this approach can inspire the study of recognition in more complex 3D systems like bilayers, liposomes, QCM, etc.

\section{Acknowledgements}

Support from the Ministry of Economy and Competitiveness of Spain is acknowledged through the following projects: CTQ2014-57515-C2 and CTQ2017-83961-R. J.J.G.-C. acknowledges the Ministry of Economy and Competitiveness for a "Ramon y Cajal" contract (\#RyC-2014-14956).

\section{Conflict of Interest}

The authors declare no conflict of interest.

\section{Appendix A. Supplementary data}

Supplementary data to this article can be found online at https:// doi.org/10.1016/j.jcis.2018.11.036.

\section{References}

[1] L. Liu, D. Xia, L.H. Klausen, M. Dong, The self-assembled behavior of DNA bases on the interface, Int. J. Mol. Sci. 15 (2014) 1901-1914, https://doi.org/10. 3390/ijms 15021901

[2] C. Avitabile, C. Diaferia, B. Della Ventura, F.A. Mercurio, M. Leone, V. Roviello, et al., Self-assembling of Fmoc-GC peptide nucleic acid dimers into 
highly fluorescent aggregates, Chem. - A Eur. J. 24 (2018) 4729-4735, https:/ doi.org/10.1002/chem.201800279.

[3] G. Zhu, Y. Xu, X. Cen, K.S. Nandakumar, S. Liu, K. Cheng, Targeting pattern-recognition receptors to discover new small molecule immune modulators, Eur. J. Med. Chem. 144 (2018) 82-92, https://doi.org/10.1016/j.ejmech.2017.12. 026.

[4] X. Shen, D.R. Corey, Chemistry, mechanism and clinical status of antisense oligonucleotides and duplex RNAs, Nucleic Acids Res. 46 (2018) 1584-1600, https://doi.org/10.1093/nar/gkx1239.

[5] J. Baillet, V. Desvergnes, A. Hamoud, L. Latxague, P. Barthélémy, Lipid and nucleic acid chemistries: combining the best of both worlds to construct advanced biomaterials, Adv. Mater. 30 (2018) 1-24, https://doi.org/10.1002/adma. 201705078.

[6] M. Angelerou, P.W.J.M. Frederix, M. Wallace, B. Yang, A. Rodger, D.J. Adams, et al., A supramolecular nucleoside-based gel: molecular dynamics simulation and characterization of its nanoarchitecture and self-assembly mechanism, Langmuir 34 (2018) 6912-6921, https://doi.org/10.1021/acs.langmuir. $8 \mathrm{~b} 00646$.

[7] M. Ramani, T.D.T. Nguyen, S. Aryal, K.C. Ghosh, R.K. Delong, Elucidating the RNA nano-bio interface: mechanisms of anticancer poly I: C RNA and zinc oxide nanoparticle interaction, J. Phys. Chem. C. 121 (2017) 15702-15710, https:// doi.org/10.1021/acs.jpcc.7b02954.

[8] J. Willem de Vries, S. Schnichels, J. Hurst, L. Strudel, A. Gruszka, M. Kwak, et al., DNA nanoparticles for ophthalmic drug delivery, Biomaterials 157 (2018) 98-106, https://doi.org/10.1016/j.biomaterials.2017.11.046.

[9] A.P. Davis, Sticking to sugars, Nature 464 (2010) 169-170.

[10] H.R. Marsden, A. Kros, Self-assembly of coiled coils in synthetic biology: inspiration and progress, Angew. Chemie - Int. Ed. 49 (2010) 2988-3005, https://doi. org/10.1002/anie.200904943.

[11] V. Amendola, G. Bergamaschi, A. Buttafava, L. Fabbrizzi, E. Monzani, Recognition and sensing of nucleoside monophosphates by a dicopper(II) cryptate, J. Am. Chem. Soc. 132 (2010) 147-156, https://doi.org/10.1021/ja9046262.

[12] R.M. Leblanc, Molecular recognition at Langmuir monolayers, Curr. Opin. Chem. Biol. 10 (2006) 529-536, https://doi.org/10.1016/j.cbpa.2006.09.010.

[13] K. Ariga, T. Nakanishi, J.P. Hill, A paradigm shift in the field of molecular recognition at the air-water interface: from static to dynamic, Soft Matter. 2 (2006) 465-477, https://doi.org/10.1039/b602732f.

[14] K. Ariga, T. Michinobu, T. Nakanishi, J.P. Hill, Chiral recognition at the air-water interface, Curr. Opin. Colloid Interf. Sci. 13 (2008) 23-30, https://doi.org/10. 1016/j.cocis.2007.08.010.

[15] T. Mori, K. Okamoto, H. Endo, J.P. Hill, S. Shinoda, M. Matsukura, et al., Mechanical tuning of molecular recognition to discriminate the single-methyl-group difference between thymine and uracil, J. Am. Chem. Soc. 132 (2010) 12868-12870, https://doi.org/10.1021/ja106653a.

[16] H. Kitano, H. Ringsdorf, Surface behaviours of nucleic acid base-containing lipids in monolayer and bilayer systems, Bull. Chem. Soc. Jpn. 58 (1985) 2826-2828.

[17] D. Berti, C. Montis, P. Baglioni, Self-assembly of designer biosurfactants, Soft Matter. 7 (2011) 7150-7158, https://doi.org/10.4013/cld.2016.142.07.

[18] H. Rosemeyer, Nucleolipids: Natural occurrence, synthesis, molecular recognition, and supramolecular assemblies as potential precursors of life and bioorganic materials, Chem. Biodivers. 2 (2005) 977-1062, https://doi.org/10.1002/ cbdv. 200590082 .

[19] C. Montis, Y. Gerelli, G. Fragneto, T. Nylander, P. Baglioni, D. Berti, Nucleolipid bilayers: a quartz crystal microbalance and neutron reflectometry study, Coll. Surf. B Biointerf. 137 (2016) 203-213, https://doi.org/10.1016/j.colsurfb. 2015.07.039.

[20] M. Ahlers, H. Ringsdorf, H. Rosemeyer, F. Seela, Orientation, recognition, and photoreaction of nucleolipids in model membranes, Coll. Polym. Sci. 268 (1990) 132-142, https://doi.org/10.1007/BF01513191.

[21] J. Huang, Y. Liang, Molecular recognition of nucleolipid amphiphile octadecanoyl ester of 1-(2-carboxyethyl) adenine to the complementary nucleobases Part I: characterization of the monolayer behavior at the air/water interface and photodimerization in the Langmuir-Blodgett, Thin Solid Films 326 (1998) 217-222, https://doi.org/10.1016/S0040-6090(98)00527-6.

[22] C. Li, J. Huang, Y. Liang, Structure control on photodimerization of Uracil and thymine moieties in nucleolipid langmuir - blodgett films by the molecular recognition effect at the air/water interface, Langmuir. 17 (2001) 2228-2234, https://doi.org/10.1021/la001105i.

[23] T. Kondo, K. Ota, Y. Matsushita, R. Kimura, T. Kawai, Molecular recognition and removal properties of Langmuir-Blodgett films of nucleolipid amphiphiles bearing thymine headgroup, Coll. Surf. A Physicochem. Eng. Asp. 273 (2006) 101-108, https://doi.org/10.1016/j.colsurfa.2005.08.012.

[24] X. Mulet, T. Kaasgaard, C.E. Conn, L.J. Waddington, D.F. Kennedy, A. Weerawardena, et al., Nanostructured nonionic thymidine nucleolipid self-assembly materials, Langmuir 26 (2010) 18415-18423, https://doi.org/10.1021/la103370q

[25] B. Desbat, N. Arazam, S. Khiati, G. Tonelli, W. Neri, P. Barthélémy, et al., Unexpected bilayer formation in langmuir films of nucleolipids, Langmuir 28 (2012) 6816-6825, https://doi.org/10.1021/la2047596.

[26] Y. Xin, X. Kong, X. Zhang, Z. Lv, X. Du, Self-assembly and molecular recognition of adenine- and thymine-functionalized nucleolipids in the mixed monolay- ers and thymine-functionalized nucleolipids on aqueous melamine at the air-water interface, Langmuir 28 (2012) 11153-11163, https://doi.org/10.1021/ la301338a.

[27] W. Lee, S. Matsika, Photochemical formation of cyclobutane pyrimidine dimers in DNA through electron transfer from a flanking base, ChemPhysChem 19 (2018) 1568-1571, https://doi.org/10.1002/cphc.201800151.

[28] M. Kanduč, A. Schlaich, A.H. De Vries, J. Jouhet, E. Maréchal, B. Demé, et al., Tight cohesion between glycolipid membranes results from balanced water-headgroup interactions, Nat. Commun. 8 (2017) https://doi.org/10.1038/ ncomms 14899 .

[29] M.N. Islam, Y. Ren, T. Kato, Polarization modulation infrared reflection absorption spectroscopy of gibbs monolayer at the air/water interface, Langmuir 18 (2002) 9422-9428, https://doi.org/10.1021/la0205708.

[30] W.P. Ulrich, H. Vogel, Polarization-modulated FTIR spectroscopy of lipid/ gramicidin monolayers at the air/water interface, Biophys. J. 76 (1999) 1639-1647, https://doi.org/10.1016/S0006-3495(99)77323-6.

[31] J. Saccani, S. Castano, F. Beaurain, M. Laguerre, B. Desbat, Stabilization of phospholipid multilayers at the air-water interface by compression beyond the collapse: A BAM PM-IRRAS, and molecular dynamics study, Langmuir 20 (2004) 9190-9197, https://doi.org/10.1021/la0489920.

[32] T.M. Nobre, F.J. Pavinatto, L. Caseli, A. Barros-Timmons, P. Dynarowicz-Łatka, O.N. Oliveira, Interactions of bioactive molecules \& nanomaterials with Langmuir monolayers as cell membrane models, Thin Solid Films 593 (2015) 158-188, https://doi.org/10.1016/j.tsf.2015.09.047.

[33] E.M. Adams, C.B. Casper, H.C. Allen, Effect of cation enrichment on dipalmitoylphosphatidylcholine (DPPC) monolayers at the air-water interface, J. Coll. Interf. Sci. 478 (2016) 353-364, https://doi.org/10.1016/j.jcis.2016.06.016.

[34] J.C. Rodrigues, L. Caseli, Incorporation of bacitracin in Langmuir films of phospholipids at the air-water interface, Thin Solid Films 622 (2017) 95-103, https:// doi.org/10.1016/j.tsf.2016.12.019.

[35] R. Wang, Y. Guo, H. Liu, Y. Chen, Y. Shang, H. Liu, The effect of chitin nanoparticles on surface behavior of DPPC/DPPG Langmuir monolayers, J. Coll. Interf. Sci. 519 (2018) 186-193, https://doi.org/10.1016/j.jcis.2018.02.021.

[36] R. Marques De Oliveira, J. Ferreira, M.J.L. Santos, R.M. Faria, O.N. Oliveira, Probing the functionalization of gold surfaces and protein adsorption by PM-IRRAS, ChemPhysChem 12 (2011) 1736-1740, https://doi.org/10.1002/cphc. 201100080

[37] R. Johann, D. Vollhardt, H. Möhwald, Study of the pH dependence of head group bonding in arachidic acid monolayers by polarization modulation infrared reflection absorption spectroscopy, Coll. Surf. A Physicochem. Eng. Asp. 182 (2001) 311-320, https://doi.org/10.1016/S0927-7757(00)00812-8.

[38] Y. Wang, X. Du, W. Miao, Y. Liang, Molecular recognition of cytosine- and guanine-functionalized nucleolipids in the mixed monolayers at the air-water interface and Langmuir-Blodgett films, J. Phys. Chem. B. 110 (2006) 4914-4923, https://doi.org/10.1021/jp055046z

[39] R.M. Silverstein, F.X. Webster, D.J. Kiemle, D.L. Bryce, Spectrometric identification of organic compounds, 8th ed., John Wiley \& Sons, New York, 2014.

[40] R.G. Snyder, H.L. Strauss, C.A. Elliger, C-H stretching modes and the structure of n-alkyl chains. 1. Long, disordered chains, J. Phys. Chem. 86 (1982) 5145-5150, https://doi.org/10.1021/j150647a002.

[41] M.D. Porter, T.B. Bright, D.L. Aliara, C.E.D. Chidsey, Spontaneously organized molecular assemblies. 4. structural characterization of «-alkyl thiol monolayers on gold by optical ellipsometry, infrared spectroscopy, and electrochemistry, Chinese J Oncol. 109 (1987) 3559-3568.

[42] Y.T. Tao, Structural comparison of self-assembled monolayers of n-alkanoic acids on the surfaces of silver, copper, and aluminum, J. Am. Chem. Soc. 115 (1993) 4350-4358, https://doi.org/10.1021/ja00063a062.

[43] H. Byrd, S. Whipps, J.K. Pike, J. Ma, S.E. Nagler, D.R. Talham, Role of the template layer in organizing self-assembled films: zirconium phosphonate monolayers and multilayers at a langmuir-blodgett template, J. Am. Chem. Soc. 116 (1994) 295-301, https://doi.org/10.1021/ja00080a034.

[44] J. Fernández, J.J. Ruiz, L. Camacho, M.T. Martin, E. Muñoz, Orientation and organization of langmuir-blodgett mixed films consisting of a phospholipid and a viologen studied by infrared spectroscopy, J. Phys. Chem. B. 104 (2000) 5573-5578, https://doi.org/10.1021/jp000474k.

[45] P. Lozano, A.J. Fernández, J.J. Ruiz, L. Camacho, M.T. Martín, E. Muñoz, Molecular organization of LB multilayers of phospholipid and mixed phospholipid/ viologen by FTIR spectroscopy, J. Phys. Chem. B. 106 (2002) 6507-6514, https: //doi.org/10.1021/jp0144505.

[46] W. Miao, X. Du, Y. Liang, Molecular recognition of nucleolipid monolayers of 1-(2-octadecyloxycarbonylethyl)cytosine to guanosine at the air-water interface and Langmuir-Blodgett films, Langmuir. 19 (2003) 5389-5396, https://doi.org/ 10.1021/la0345690.

[47] S.L. Mayo, B.D. Olafson, W.A. Goddard, DREIDING: a generic force field for molecular simulations, J. Phys. Chem. 94 (1990) 8897-8909, https://doi.org/10. 1021/j100389a010.

[48] D. Vlachakis, E. Bencurova, N. Papangelopoulos, S. Kossida, Current state-of-the-art molecular dynamics methods and applications, In: Adv. Protein Chem. Struct. Biol., Elsevier, 2014, pp. 269-313. 
[49] M. Edelwirth, J. Freund, S.J. Sowerby, W.M. Heckl, Molecular mechanics study of hydrogen bonded self-assembled adenine monolayers on graphite, Surf. Sci. 417 (1998) 201-209, https://doi.org/10.1016/S0039-6028(98)00576-7.

[50] K. Sfyrakis, A. Provata, D.C. Povey, B.J. Howlin, Local sequential minimization of double stranded B-DNA using Monte Carlo annealing, J. Mol Model. 10 (2004) 185-197, https://doi.org/10.1007/s00894-004-0182-0.

[51] J. He, T.a. Kaprak, M.a. Jones, T.D. Lash, Normal and abnormal heme biosynthesis Part 4: molecular dynamics simulations of coproporphyrinogen-III and related di- and tricarboxylic acids, J. Porphyr. Phthalocyanines 9 (2005) 170-185, https://doi.org/10.1142/S1088424605000241.

[52] M. Pérez-Morales, J.M. Pedrosa, M.T. Martín-Romero, D. Möbius, L. Camacho, T. Martín-Romero, et al., Reversible trilayer formation at the air - water interface from a mixed monolayer containing a cationic lipid and an anionic porphyrin, $\mathrm{J}$. Phys. Chem. B. 108 (2004) 4457-4465, https://doi.org/10.1021/jp037282k.

[53] C. Rubia-Payá, E. Jimenez-Millán, J.J. Giner-Casares, G. Brezesinski, M.T Martín-Romero, L. Camacho, From two-dimensional to three-dimensional at the air/water interface: the self-aggregation of the acridine dye in mixed monolayers, Langmuir 29 (2013) 4796-4805, https://doi.org/10.1021/la400483d.
[54] H. Mohwald, Phospholipid and phospholipid-protein monolayers at the air/water interface, Annu. Rev. Phys. Chem. 41 (1990) 441-476, https://doi.org/10.1146/ annurev.pc.41.100190.002301.

[55] G. De Miguel, M.T. Martín-Romero, J.M. Pedrosa, E. Muñoz, M.

Pérez-Morales, T.H. Richardson, et al., Improvement of optical gas sensing using LB films containing a water insoluble porphyrin organized in a calixarene matrix, J. Mater. Chem. 17 (2007) 2914-2920, https://doi.org/10.1039/ b701811h.

[56] J.J. Giner-Casares, M. Pérez-Morales, H.J. Bolink, E. Muñoz, G. de Miguel, M.T. Martín-Romero, et al., Molecular organization of a water-insoluble iridium(III) complex in mixed monolayers, J. Coll. Interf. Sci. 315 (2007) 278-286, https://doi.org/10.1016/j.jcis.2007.06.015.

[57] J.J. Giner-Casares, M. Pérez-Morales, H.J. Bolink, N. Lardiés, E. Muñoz, G. De Miguel, et al., Segregation of lipid in Ir-dye/DMPA mixed monolayers as strategy to fabricate 2D supramolecular nanostructures at the air-water interface, J. Mater. Chem. 18 (2008) 1681-1688, https://doi.org/10.1039/b719910d. 